\title{
A Comprehensive Systematic Review and Meta-analysis on the Risk Factors of Stroke in Iranian Population
}

\author{
Reza Tabrizi, PhD'; Kamran B Lankarani, MD²; Bahareh Kardeh, MD³; Hamed Akbari, MSc ${ }^{4,5}$; Mahmoud Reza Azarpazhooh, MD ${ }^{6,7,8}$; Afshin \\ Borhani-Haghighi, MD $^{3^{*}}$ \\ ${ }^{1}$ Non-Communicable Diseases Research Center, Fasa University of Medical Sciences, Fasa, Iran \\ ${ }^{2}$ Health Policy Research Center, Institute of Health, Shiraz University of Medical Sciences, Shiraz, Iran \\ ${ }^{3}$ Clinical Neurology Research Center, Shiraz University of Medical Sciences, Shiraz, Iran \\ ${ }^{4}$ Department of Biochemistry, School of Medicine, Kerman University of Medical Sciences, Kerman, Iran \\ ${ }^{5}$ Student Research Committee, School of Medicine, Kerman University of Medical Sciences, Kerman, Iran \\ ${ }^{6}$ Department of Neurology, Ghaem Hospital, Mashhad University of Medical Sciences, Mashhad, Iran \\ ${ }^{7}$ Department of Clinical Neurological Science, University Hospital, London Health Science Center, University of Western Ontario, London, \\ Ontario, Canada \\ ${ }^{8}$ Department of Epidemiology and Biostatistics, Western University, London, Ontario, Canada
}

\begin{abstract}
Background: There are limited data on vascular risk factors (VRFs) in low- and middle-income countries (LMICs). This meta-analysis was completed to summarize the existing evidence on stroke risk factors (SRFs) in the Iranian population.

Methods: An electronic literature search of the databases including PubMed, Embase, Web of Science, Scopus, Scientific Information Database (SID), Magiran, and IranMedex was performed to identify the related articles published up to March 2018. For categorical or continuous variables, the data were also pooled using the fixed- or the random-effect models, respectively, expressed as odds ratio (OR) or weighted mean difference (WMD).

Results: A total of 15 articles were recruited. The risk of stroke was associated with mean age, but not gender. Among traditional VRFs, hypertension (HTN), systolic and diastolic blood pressure (DBP), diabetes mellitus (DM), and fasting blood glucose (FBG) were associated with increased risk of stroke. Apart from the high circulating levels of triglycerides (TG), low-density lipoproteincholesterol (LDL-C), total cholesterol (TC), and low high-density lipoprotein-cholesterol (HDL-C), other potential risk factors namely cigarette smoking (CS), opioid addiction (OD), and waist circumference (WC) were identified to be independent stroke determinants.

Conclusion: The present systematic review and meta-analysis provided a summary of the most important SRFs, which are potentially modifiable and preventable. Overall, Iran, similar to many other LMICs, is experiencing an ever-increasing rate of stroke-prone elderly people. The LMICs are thus suggested to develop national approaches to recognize and address VRFs, to monitor and control CS and OD rates, and to encourage a healthy lifestyle.

Keywords: Iran, Low- and middle-income countries, Meta-analysis, Risk factor, Stroke

Cite this article as: Tabrizi R, Lankarani $\mathrm{KB}$, Kardeh B, Akbari H, Azarpazhooh MR, Borhani-Haghighi A. A comprehensive systematic review and meta-analysis on the risk factors of stroke in Iranian population. Arch Iran Med. 2021;24(1):64-77. doi: 10.34172/aim.2021.10.
\end{abstract}

Received: May 30, 2020, Accepted: October 4, 2020, ePublished: January 1, 2021

\section{Introduction}

Stroke, with its main types including ischemic and hemorrhagic, is known as the second leading cause of death and the fourth leading cause of mortality worldwide; thus, approximately 5.7 million deaths are recorded annually due to this medical condition, accounting for $9.7 \%$ of all-cause mortality across the world. ${ }^{1,2}$ It also occurs in $1-3$ cases per thousand people each year in developed countries, ${ }^{3}$ and it is growing in Iran, affecting 372 people per 100000 population. ${ }^{4}$ The reports in this respect have also demonstrated that age-standardized stroke mortality have totally decreased in the past two decades. However, the absolute number of individuals experiencing a stroke every year, as well as stroke survivors, related deaths, and the overall global burden of this condition (i.e., the disability-adjusted life years: DALYs lost) have been great and increasing, especially in low- and middle-income countries (LMICs) over the past decades. ${ }^{5}$ The LMICs also account for more than $85 \%$ of strokes correlated with mortality and morbidity, leading to the highest disease burden in these countries. In addition, geographic variations can add to the epidemiology of the stroke, as the highest incidence and prevalence rates are being reported in the same nations. ${ }^{6}$

Determining potential stroke risk factors (SRFs) within each geographical and ethnic region is accordingly of 
utmost importance to adjust stroke prevention programs based on existing needs and facilities. ${ }^{7,8}$ Previously, it had been reported that there could be regional variations in the significance of risk factors, which had in turn resulted in worldwide discrepancies observed in incidence rate and subtypes of stroke. ${ }^{9}$ Recently, the INTERSTROKE, a large-scale case-control study of SRFs in 32 countries, has reported 10 potentially modifiable risk factors for either ischemic or hemorrhagic stroke, associated with $90 \%$ population attributable risks of stroke in each major geographical region of the world as well as in specific age and gender groups. ${ }^{10,11}$ The regional variations of SRFs are thus of great importance for risk stratification and lifestyle modification programs in each region. In this way, analysis of SRFs should be conducted in different regional and ethnic groups in order to specify stroke prevention programs and increase their success rates. ${ }^{2}$

Iran, as a populous LMIC located in the Middle East with considerably diverse ethnic groups and social classes, largely reflects the core of such countries. In addition, the relatively younger age of stroke in Iran (approximately one decade earlier in Iran than that in Western countries) can significantly increase stroke DALYs. ${ }^{12}$ Thus, providing better evidence on SRFs in the Iranian population as an LMIC is particularly relevant. Although several studies have previously addressed the analysis of SRFs in the Iranian population, there are variations in methodologies and sample sizes, restricting their implications to develop a nationwide stroke prevention program based on a risk stratification model. ${ }^{13-19}$ With this purpose in mind and given the unavailability of structured studies, pooling literature data, the present systematic review and metaanalysis was undertaken to determine SRFs in the Iranian population.

\section{Materials and Methods}

This meta-analysis was designed and performed according to the guidelines in the preferred reporting items for systematic reviews and meta-analyses (PRISMA).

\section{Search Strategy}

As the main strategy, two authors independently searched international and national databases including PubMed, Embase, Web of Science, Scopus, Scientific Information Database (SID), Magiran, and IranMedex for relevant articles published until March 2018. The searches were also conducted using the following $\mathrm{MeSH}$ terms and text words as well as their Persian equivalents: ["stroke" OR "brain infarction" OR "ischemic stroke" OR "intracerebral hemorrhagic' OR “intracranial bleed” OR "hemorrhagic stroke" OR "subarachnoid hemorrhage" OR "cerebrovascular disorders" OR "cerebrovascular disease"] AND ["all related risk factors" (such as demographic, anthropometric, vascular, diabetes mellitus, hypertension, cigarette smoking, opioid dependence, and hyperlipidemia factors)] AND ["Iran" OR "Iranian"].

In order to augment the sensitivity of the search strategy, the reference lists of the included articles were manually screened out to detect any additional relevant studies and even Google Scholar as a gray literature was hand-scanned. The searches were restricted to observational studies published in the English or Persian literature without any limitations in their publication date.

\section{Definitions}

Most of the investigated SRFs had similar definitions in national and international publications. However, the authors used the definitions of SRFs across selected articles as included here: hypertension (HTN) HTN represented systolic blood pressure $(\mathrm{SBP}) \geq 140 \mathrm{~mm} \mathrm{Hg}$ and/or diastolic blood pressure (DBP) $\geq 90 \mathrm{~mm} \mathrm{Hg}$ or taking anti-hypertensive medications. ${ }^{20}$ Diabetes mellitus (DM) was defined as fasting blood glucose (FBG) $\geq 7$ $\mathrm{mmol} / \mathrm{L}, 2 \mathrm{~h}-\mathrm{PG} \geq 11.1 \mathrm{mmol} / \mathrm{L}$ or current intake of antidiabetic medicines. ${ }^{20}$ Cigarette smoking (CS) represented current or past use of cigarettes or any other type of pipes or smoking instruments, ${ }^{20}$ and family history of cardiovascular diseases (CVDs) was described as any prior diagnosis of CVDs in first-degree relatives. ${ }^{20}$ Also, body mass index (BMI) was weight divided by height squared $\left(\mathrm{kg} / \mathrm{m}^{2}\right)$ and waist circumference (WC) was measured in a standing position at the point midway between the lowest rib and the iliac crest in both genders ${ }^{21}$. Opioid dependence (OD) was defined according to the Diagnostic and Statistical Manual of Mental Disorders, fourth edition, text revision (DSM-IV-TR) (304.00, opium dependence): continued for at least one year (via inhalation or oral ingestion). ${ }^{22}$ Furthermore, total-cholesterol (TC), highdensity lipoprotein-cholesterol (HDL-C), low-density lipoprotein-cholesterol (LDL-C), triglycerides (TG), and FBG levels in serum were further considered.

Inclusion and Exclusion Criteria

Studies meeting the eligibility criteria including observational human studies with cross-sectional, casecontrol, or cohort designs as well as articles investigating associations between related risk factors and stroke were selected for this study. Moreover, studies published until March 2018, without any limitations on their sample size, were found eligible. We used articles reporting sufficient data to extract the number of events for calculating odds ratios (ORs) or weighted mean differences (WMDs) with 95\% confidence intervals (CIs) for the related risk factors in patients with first-ever stroke, compared with control groups without any strokes or related CVDs. However, in-vitro studies, animal experiments, case reports, review articles, and seminar abstracts with no full-texts, together with studies that had not obtained the least required scores of the quality assessment process were excluded. 
Data Extraction and Quality Assessment

Two independent authors (RT and BK) extracted the data from the retrieved articles and imported them into standard forms in the Microsoft Excel spreadsheets. Accordingly, the extracted information included first author's name, date published, study setting, participants' demographic characteristics, sample size (either in stroke patients or control group), study design, stroke type, risk factors, and required data to estimate ORs or WMDs and 95\% CIs for risk factors if they were categorical or continuous variables, respectively, in stroke patients and control groups. The quality of the given studies was also assessed by two independent authors (RT and BK) according to a comprehensive quality assessment using the NewcastleOttawa Scale, comprised of eight and six items for cohort/ case-control and cross-sectional studies, respectively. These items had covered three aspects including selection, comparability, and exposure. The selected study obtaining 4-6 points could be considered as an article of moderate quality and the one with more than 6 points could be assigned as high quality.

In case of disagreements in the domains of data extraction and quality assessment, RT and BK discussed to reach consensus or resolved the disagreement through talks with a third author (A-BH).

\section{Data Synthesis and Statistical Analysis}

All the statistical analyses were performed using the Stata (version 12.0) (Stata Corp., College Station, TX) software package. The association between the related risk factors and the risk of stroke was also estimated. To assess the overall pooled effect sizes (ESs), unadjusted ORs or WMDs were respectively employed with 95\% CIs for continuous or categorical factors. The Cochran $\mathrm{Q}$ test and the I-squared $\left(\mathrm{I}^{2}\right)$ test were further utilized to measure heterogeneity across the included studies. According to the heterogeneity findings, whenever $\mathrm{I}^{2} \geq 50 \%$ and $P<0.05$, a random-effect model using the DerSimonian and Laird method could be applied to combine the studies, a fixedeffect model could be also implemented using the Mantel and Haenszel method provided that $\mathrm{I}^{2}<50 \%$ and $P>$ 0.05 . The sensitivity analysis was additionally conducted to examine the impact of each study on the overall pooled ESs after excluding each one using the leave-oneout method. Subgroup analyses were also completed to identify the possible sources of heterogeneity regarding the following moderator variables: type of controls (healthy vs. hospital-based control), type of stroke (all vs. ischemic vs. hemorrhagic stroke), study design (cross-sectional vs. cohort vs. case-control study), and matching status (nonmatching vs. gender or age matching). The Begg's and the Egger's tests were consequently utilized to evaluate the possibility of publication bias. The two-tailed $P \otimes 0.05$ was also considered as statistically significant.

\section{Results}

Characteristics of Selected Studies

In the first step searching the online databases, 573 articles related to SRFs were identified. After removing the duplicates and the irrelevant ones, 15 studies (namely, 10 case-control, three cohort, and two cross-sectional studies) were selected for the current meta-analysis. The flowchart details of the systematic process of study identification and selection are summarized in Figure 1. The sample size in the selected studies included 13342 participants (10009 ischemic, 255 hemorrhagic, and 3078 all stroke) ranging from 72 to 5398 cases. The selected articles had been published from 2003 up to March 2018. The characteristics of the observational studies included are presented in Table 1.

\section{Main Outcomes}

Forest plots reporting the meta-analysis of the included studies on SRFs consisting of demographic, traditional vascular, and life-style factors are illustrated in Figures 2 and 3 .

The pooled findings showed that 13 factors influenced the risk of stroke in the Iranian population. As compared with the control group, the risk of stroke was correlated with the mean age (WMD $=5.49$ years; 95\% CI, 4.45, 6.56; $\left.P<0.001 ; \mathrm{I}^{2}: 45.4 \%\right)$ of the patients. Nevertheless, there was no significant relationship between gender (OR $\left.=1.23 ; 95 \% \mathrm{CI}, 0.85,1.79 ; P=0.272 ; \mathrm{I}^{2}: 65.8 \%\right)$ and risk of stroke.

Among the traditional vascular risk factors (VRFs), HTN $\left(\mathrm{OR}=3.56 ; 95 \% \mathrm{CI}, 3.00,4.23 ; P<0.001 ; \mathrm{I}^{2}\right.$ : $37.7 \%$ ), SBP (WMD = $13.42 \mathrm{mmHg}$; $95 \% \mathrm{CI}, 10.19$, 16.64; $\left.P<0.001 ; \mathrm{I}^{2}: 0.0 \%\right), \mathrm{DBP}(\mathrm{WMD}=6.66 \mathrm{~mm} \mathrm{Hg}$; 95\% CI, 4.61, 8.70; $\left.P<0.001 ; \mathrm{I}^{2}: 0.0 \%\right)$, and DM (OR = 2.15; 95\% CI, 1.41, 3.29; $\left.P<0.001 ; \mathrm{I}^{2}: 74.4 \%\right)$ were the ones increasing the risk of stroke. Also, high levels of FBG (WMD = 36.86 mg/dL; 95\% CI, 7.66, 66.07; $P=0.013$; $\left.\mathrm{I}^{2}: 89.5 \%\right)$, TG (WMD = $21.48 \mathrm{mg} / \mathrm{dL} ; 95 \% \mathrm{CI}, 10.03$, 32.94; $\left.P<0.001 ; \mathrm{I}^{2}: 60.9 \%\right), \mathrm{TC}(\mathrm{WMD}=15.08 \mathrm{mg} / \mathrm{dL}$; 95\% CI, 0.48, 29.68; $\left.P=0.043 ; \mathrm{I}^{2}: 86.9 \%\right)$, LDL-C levels $(\mathrm{WMD}=23.89 \mathrm{mg} / \mathrm{dL} ; 95 \% \mathrm{CI}, 0.93,46.84 ; P=0.041$; $\left.I^{2}: 94.9 \%\right)$, and low levels of HDL-C (WMD = -2.31 mg/ dL; 95\% CI, $-4.30,-0.33 ; P=0.023$; I : 14.24\%) were other factors affecting the risk of stroke. Based on two included articles, no significant association was found between family history of CVDs (OR $=1.94 ; 95 \% \mathrm{CI}$, $\left.0.94,4.06 ; P=0.077 ; \mathrm{I}^{2}: 51.1 \%\right)$ and risk of stroke. Lifestyle factors including $\mathrm{OD}(\mathrm{OR}=3.00 ; 95 \% \mathrm{CI}, 1.81$, 4.98; $\left.P<0.001 ; \mathrm{I}^{2}: 0.0 \%\right)$ and $\mathrm{CS}(\mathrm{OR}=1.44 ; 95 \% \mathrm{CI}$, $\left.1.02,2.03 ; P=0.038 ; \mathrm{I}^{2}: 64.0 \%\right)$ had further augmented the risk of stroke. However, WC (WMD = 3.25 CM; 95\% CI, 1.44, 5.06; $\left.P<0.001 ; \mathrm{I}^{2}: 27.7 \%\right)$ was associated with stroke. There was also no significant relationship between BMI (WMD $=0.05 \mathrm{~kg} / \mathrm{m}^{2} ; 95 \% \mathrm{CI},-1.41,1.50 ; P=$ $0.949 ; \mathrm{I}^{2}: 79.9 \%$ ) and risk of stroke (Table 2). 


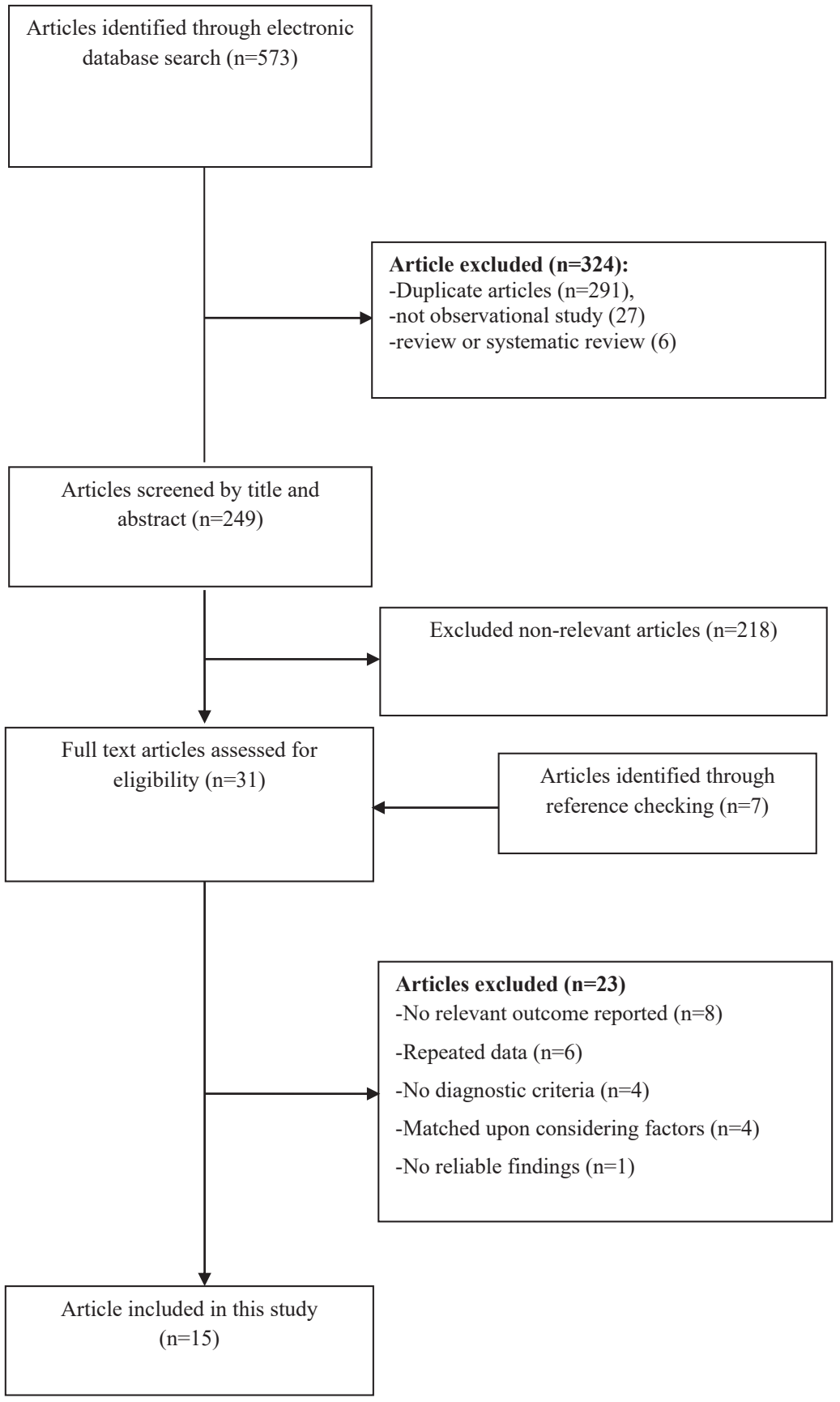

Figure 1. Literature Search and Review Flowchart for Selection of Studies.

Subgroup and Sensitivity Analyses

Subgroup analyses were conducted to identify the effects of moderator variables, i.e. type of control, stroke, study design, and matching status on heterogeneity statistics. The reduction of heterogeneity was demonstrated in some strata of the subgroups as shown in Table 2.

In subgroup analyses for gender, a significant association was observed between male gender and increased risk of stroke in studies with all types of stroke $(\mathrm{OR}=1.71$, 95\% CI: 1.26, 2.33) and the ones with non-matching participants (OR $=1.39,95 \% \mathrm{CI}: 1.09,1.77)$, compared with other groups. With regard to CS and risk of stroke, there was also a significant relationship in studies with all types of stroke $(\mathrm{OR}=2.72,95 \% \mathrm{CI}: 1.75,4.24)$ and crosssectional ones (OR $=2.25,95 \%$ CI: 1.19, 4.23) vs. studies with other strata. The findings correspondingly indicated a significant rising trend in TG levels in patients with stroke in studies with healthy control (WMD $=24.48 \mathrm{mg} /$ dL; 95\% CI 10.15, 38.81) and case-control ones (WMD $=23.99 \mathrm{mg} / \mathrm{dL} ; 95 \%$ CI 7.46, 40.51), in comparison with other strata. Considering TC levels, there was a significant growth in patients with stroke in case-control studies with gender or age matching (WMD $=21.23 \mathrm{mg} / \mathrm{dL} ; 95 \% \mathrm{CI}$ $2.16,40.30)$, compared with other strata. There was also a significant association in terms of the increased risk of stroke and LDL-C levels (WMD = $41.63 \mathrm{mg} / \mathrm{dL} ; 95 \%$ 


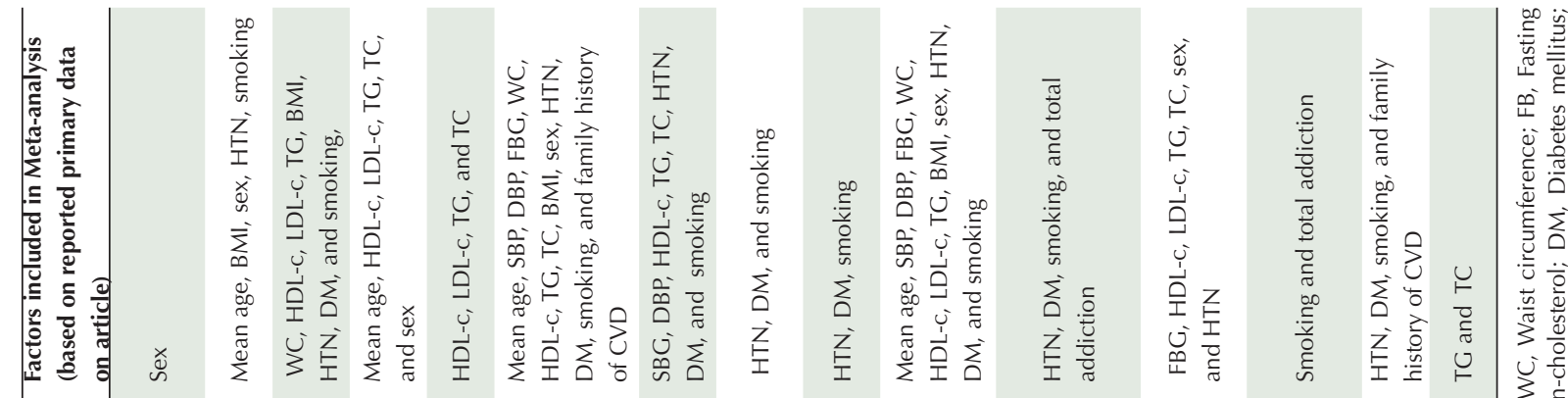

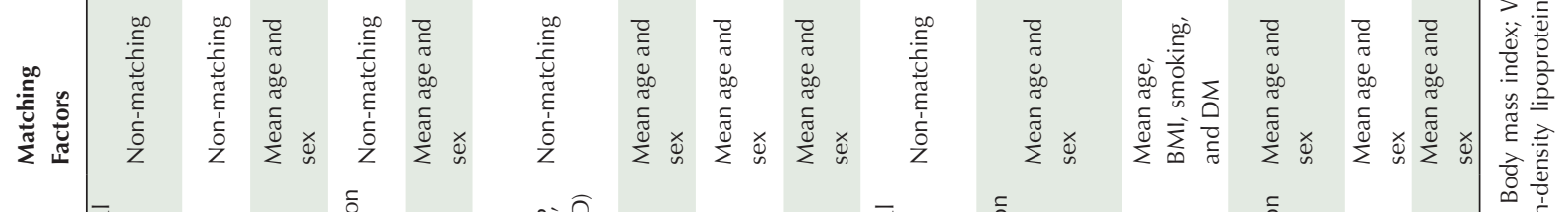

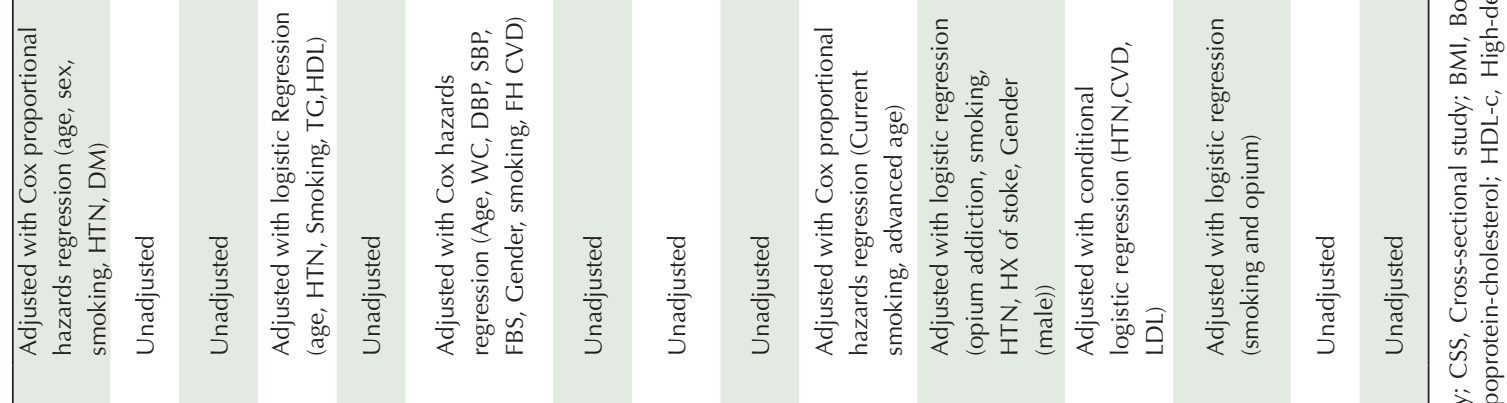

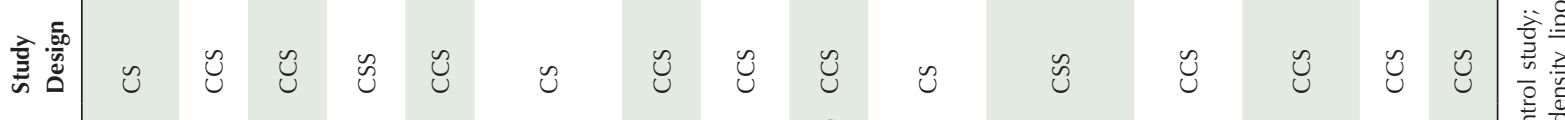

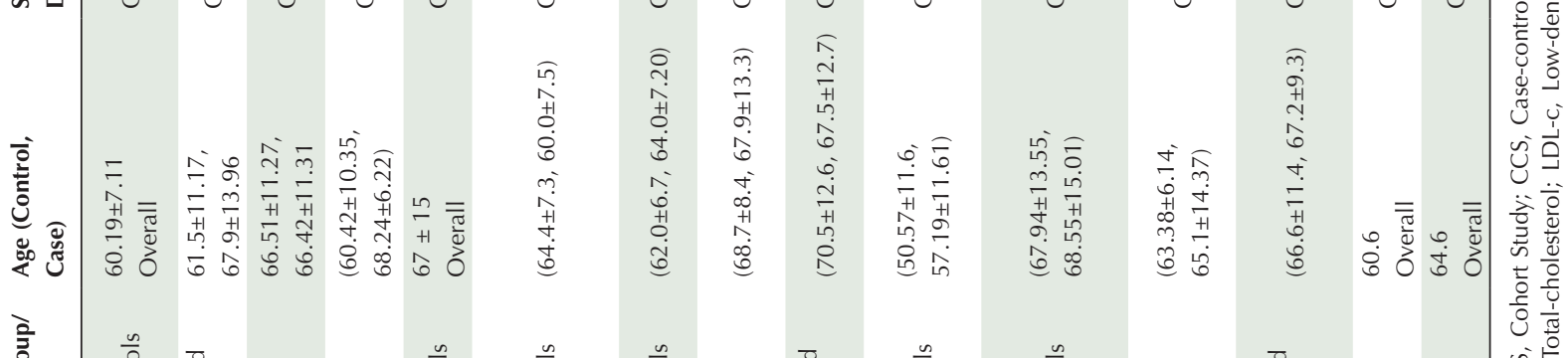

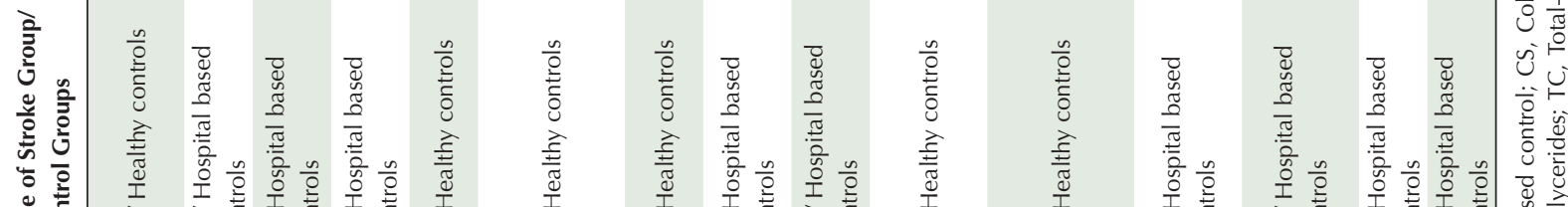

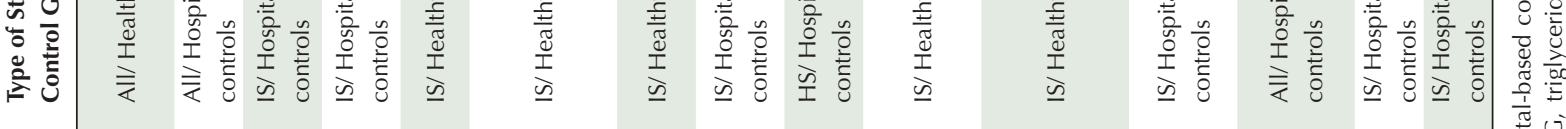

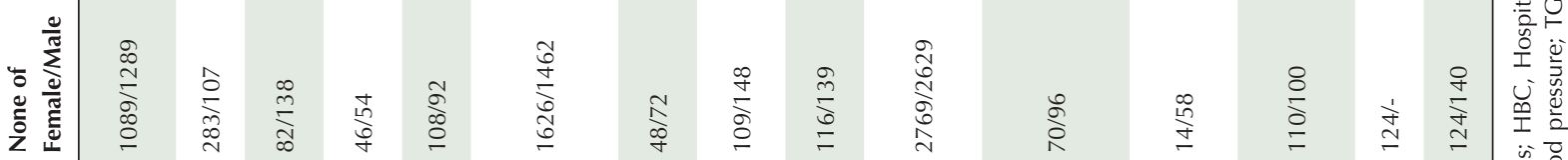

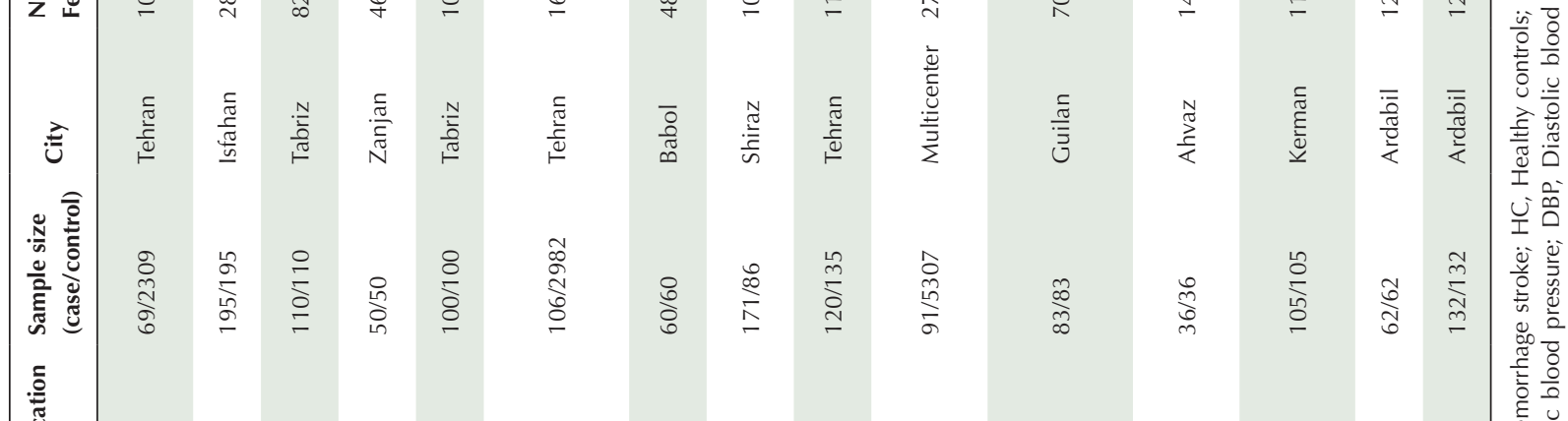

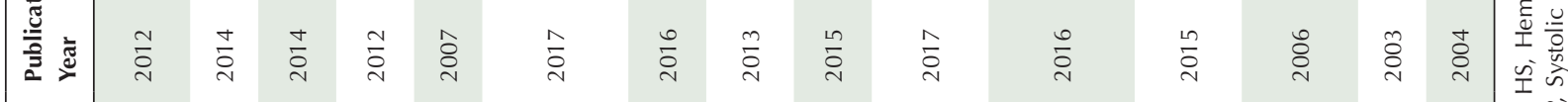

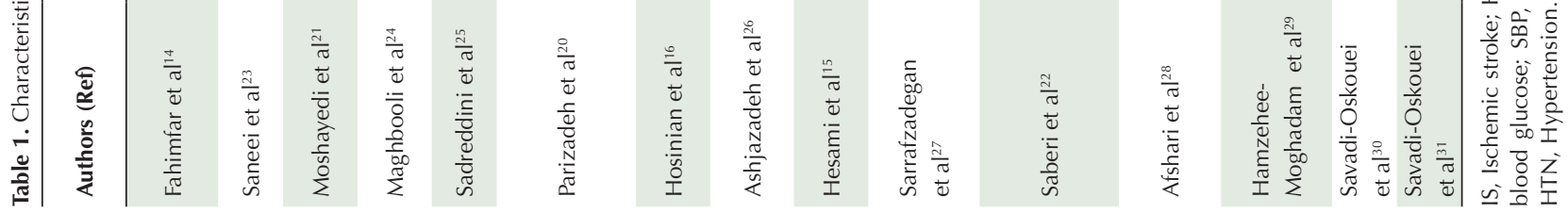




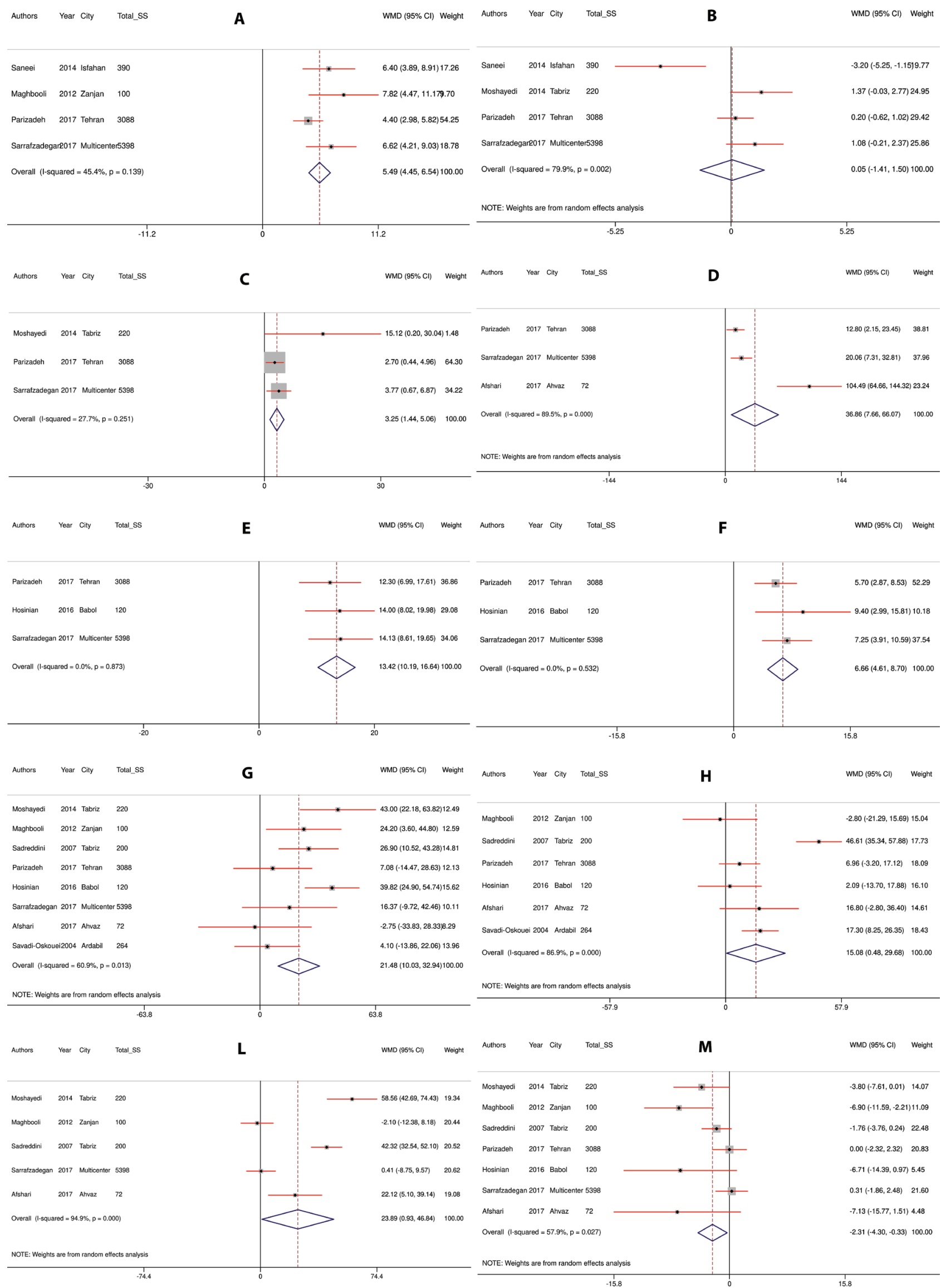

Figure 2. Meta-analysis of Stroke Factors Using Weighted Mean Differences Estimates for A) mean Age, B) BMI, C) WC, D) FBG, E) SBP, F) DBP, G) TG, H) TC, L) LDL-C, and M) HDL-C in the Stroke Patients and Control Groups (Cl = 95\%). 

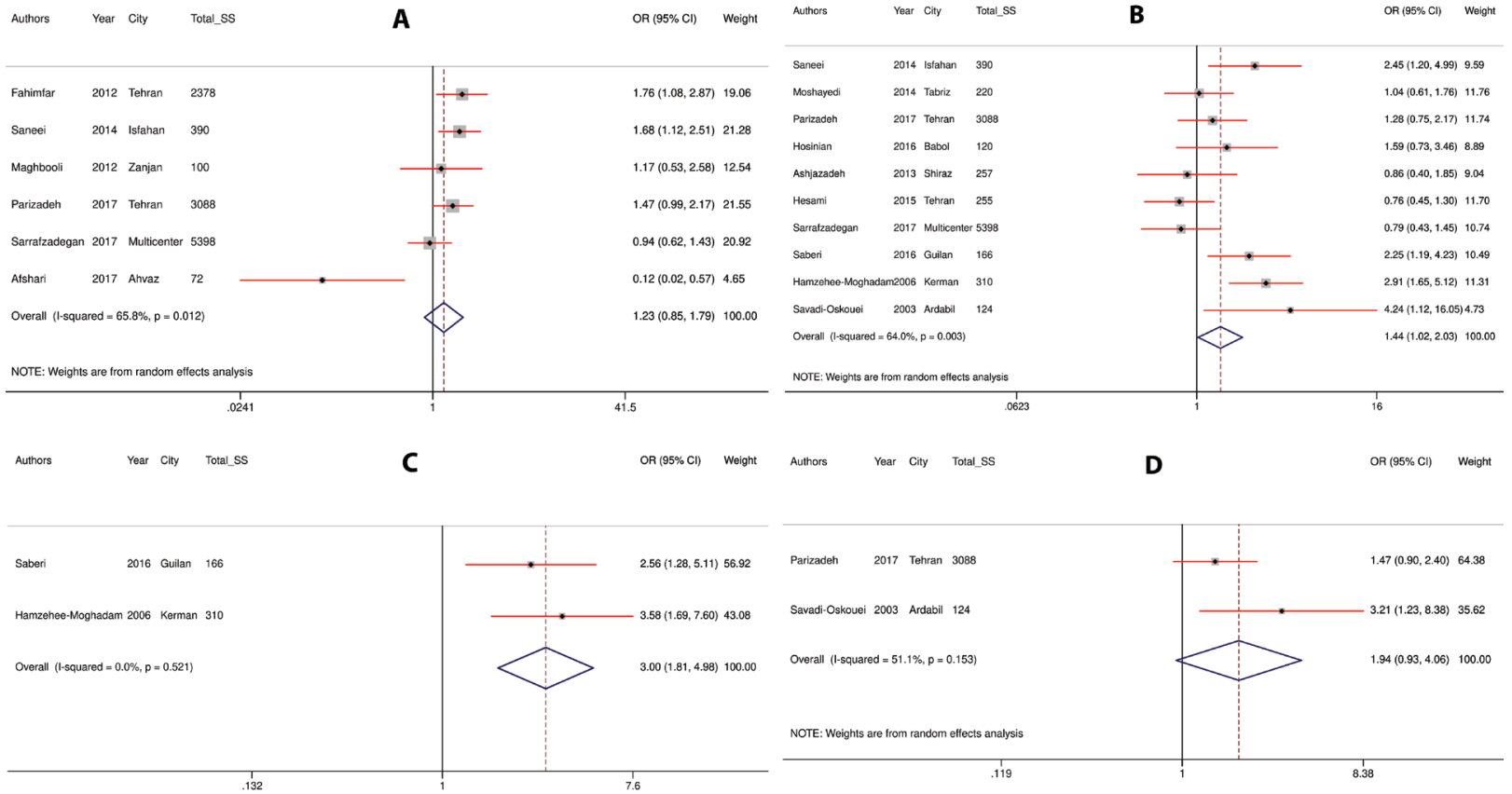

Authors Year City Total_ss

E

Moshayedi 2014 Tabriz 220

Parizadeh $\quad 2017$ Tehran 3088

Hosinian $\quad 2016$ Babol $\quad 120$

Ashjazadeh 2013 Shiraz 257

Hesami 2015 Tehran 255

Sarrafzadegan 2017 Multicenter 5398

Saberi 2016 Guilan 166

Savad-Oskouei 2003 Arrabil 124

Overall (1.-squared $=74.4 \%, \rho=0.000$ )

NOTE: Weights are trom random eftects andys

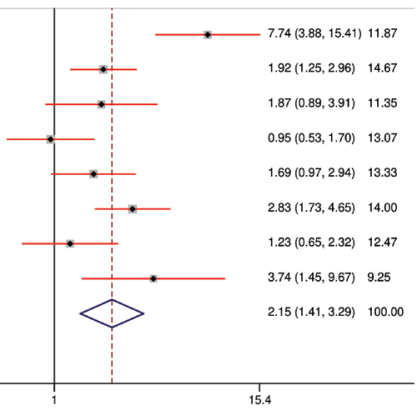

OR $(95 \%$ CI) Weight Authors Year City Total_SS

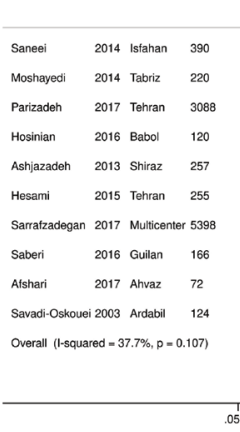

OR $(95 \%$ CI) Weight $3.07(1.77,5.33) \quad 10.51$ $2.28(1.53,3.39) \quad 23.61$ $4.07(1.89 .8 .75) 4.73$ $3.79(2.17,6.61) 9.47$ $4.66(2.63,8.25) \quad 8.27$ $3.03(2.00,4.60) \quad 16.80$ $2.88(1.50,5.55) 7.72$ $3.50(1.26,9.72) 2.91$ $8.38(3.73,18.83) 2.95$ $3.56(3.00,4.23) \quad 100.00$

Figure 3. Meta-analysis of Stroke Factors Using Odds Ratios (ORs) Estimates for A) Sex, B) Smoking, C) Opioid Dependency, D) Family Hx of CVD, E) DM, and F) HTN in the Stroke Patients and Control Groups ( $\mathrm{Cl}=95 \%)$.

CI 23.63, 58.93). For low HDL-C levels, there was a significant decrease in patients with stroke in studies with hospital-based controls (WMD $=-5.25 \mathrm{mg} / \mathrm{dL} ; 95 \% \mathrm{CI}$ $-8.05,-2.45)$, the ones designed with case-control groups, and gender or age matching (WMD $=-2.77 \mathrm{mg} / \mathrm{dL} ; 95 \%$ CI -4.67, -0.87) in comparison with other strata.

After excluding several studies from the sensitivity analysis, the results revealed that the relationship between gender, CS, FBG, TC, LDL-C, and low levels of HDL-C and the risk of stroke had changed significantly upon respectively excluding the articles by Afshari et $\mathrm{al},{ }^{28}$ Hamzehee-Moghadam et $\mathrm{al},{ }^{29}$ Parizadeh et al, ${ }^{20}$ SavadiOskouei et al, ${ }^{31}$ Moshayedi et al, ${ }^{21}$ and Maghbooli et al. ${ }^{24}$ The association between CS, FBG, and HDL-C levels and the risk of stroke also changed significantly after removing more than one study.

For the remaining factors, there was no significant change between the pre- and post-sensitivity WMDs or ORs. The lower and the higher pooled WMDs or ORs in the sensitivity analyses are presented in Table 3.
Publication Bias and Quality Assessment

The Begg's and the Egger's tests showed that publication bias had affected only four factors in the studies on the Iranian population, which included mean age $(P$ Begg's test $=0.17, P$ Egger's test $<0.01)$, FBG levels $\left(P_{\mathrm{Bg}}=0.11\right.$, $\left.P_{\mathrm{Ee}}=0.01\right)$, WC $\left(P_{\mathrm{Bg}}=0.11, P_{\mathrm{Ee}}=0.04\right)$, and low levels of HDL-C $\left(P_{\mathrm{Bg}}=0.09, P_{\mathrm{Ee}}=0.02\right)$. Non-parametric methods (i.e., Duval and Tweedie' Trim and Fill method) were further utilized to compute the findings of the removed observational studies for two factors; however, the pooled ESs for the related factors did not change significantly after using the method.

Likewise, quality assessment using the NewcastleOttawa Scale established that $86.67 \%$ of the selected primary studies obtained at least two stars for selection, $100 \%$ of these articles were assigned with at least one star for comparability, and $93.34 \%$ of the primary studies included were given at least two stars for exposure category. The detailed quality assessment results are summarized in Table 4. 
Table 2. Results of Overall and Subgroup Meta-analyses for Estimating the Association Between Risk Factors and Stroke

\begin{tabular}{|c|c|c|c|c|c|c|c|}
\hline Variables & & & $\mathbf{K}^{*}$ & $I^{2}(\%)$ & $Q$ test & Pooled ES $(95 \% \mathrm{CI})$ & $P$ Value \\
\hline Mean age (year) & Total & & 4 & 45.4 & 5.49 & $5.49(4.45,6.56)^{\mathrm{WMD}}$ & $<0.001$ \\
\hline \multirow{11}{*}{ Sex (male vs. female) } & Total & & 6 & 65.8 & 14.60 & $1.23(0.85,1.79)^{\mathrm{OR}}$ & 0.272 \\
\hline & \multirow{2}{*}{ Type of control } & Healthy controls & 3 & 52.0 & 4.17 & $1.33(0.93,1.90)^{\mathrm{OR}}$ & 0.118 \\
\hline & & Hospital-based controls & 3 & 80.9 & 10.49 & $0.81(0.27,2.39)^{\mathrm{OR}}$ & 0.701 \\
\hline & \multirow{3}{*}{ Type of stroke } & All & 2 & 0.0 & 0.02 & $1.71(1.26,2.33)^{\mathrm{OR}}$ & 0.001 \\
\hline & & IS & 4 & 71.0 & 10.35 & $0.94(0.53,1.67)^{\mathrm{OR}}$ & 0.824 \\
\hline & & $\mathrm{HS}$ & - & - & - & - & - \\
\hline & \multirow{3}{*}{ Study design } & Cohort & 3 & 52.0 & 4.17 & $1.33(0.93,1.90)^{\mathrm{OR}}$ & 0.118 \\
\hline & & Case-control & 2 & 90.3 & 10.34 & $0.50(0.40,6.84)^{\mathrm{OR}}$ & 0.602 \\
\hline & & Cross-sectional & 1 & - & 0.00 & $1.17(0.53,2.58)^{\mathrm{OR}}$ & 0.688 \\
\hline & \multirow{2}{*}{ Matching } & Non-matching & 5 & 25.6 & 5.38 & $1.39(1.09,1.77)^{\mathrm{OR}}$ & 0.007 \\
\hline & & Age matching & 1 & - & 0.00 & $0.12(0.02,0.57)^{\mathrm{OR}}$ & 0.008 \\
\hline \multirow{11}{*}{ Smoking (Yes vs. No) } & Total & & 10 & 64.0 & 24.97 & $1.44(1.02,2.03)^{\mathrm{OR}}$ & 0.038 \\
\hline & \multirow{2}{*}{ Type of control } & Healthy controls & 4 & 47.5 & 5.72 & $1.35(0.88,2.09)^{\mathrm{OR}}$ & 0.172 \\
\hline & & Hospital-based controls & 6 & 74.0 & 19.22 & $1.53(0.89,2.62)^{\mathrm{OR}}$ & 0.124 \\
\hline & \multirow{3}{*}{ Type of stroke } & All & 2 & 0.0 & 0.14 & $2.72(1.75,4.24)^{\mathrm{OR}}$ & $<0.001$ \\
\hline & & IS & 7 & 42.9 & 10.52 & $1.29(0.92,1.82)^{\mathrm{OR}}$ & 0.136 \\
\hline & & $\mathrm{HS}$ & 1 & - & 0.00 & $0.76(0.45,1.30)^{\mathrm{OR}}$ & 0.325 \\
\hline & \multirow{3}{*}{ Study design } & Cohort & 2 & 28.0 & 1.39 & $1.03(0.64,1.65)^{\mathrm{OR}}$ & 0.915 \\
\hline & & Case-control & 7 & 68.9 & 19.31 & $1.53(0.96,2.43)^{\mathrm{OR}}$ & 0.075 \\
\hline & & Cross-sectional & 1 & - & 0.00 & $2.25(1.19,4.23)^{\mathrm{OR}}$ & 0.012 \\
\hline & \multirow{2}{*}{ Matching } & Non-matching & 3 & 64.6 & 5.65 & $1.32(0.73,2.39)^{\mathrm{OR}}$ & 0.365 \\
\hline & & Sex or age matching & 7 & 68.5 & 19.06 & $1.51(0.96,2.38)^{\mathrm{OR}}$ & 0.075 \\
\hline Opioid dependency (Yes vs. No) & Total & & 2 & 0.00 & 0.41 & $3.00(1.81,4.98)^{\mathrm{OR}}$ & $<0.001$ \\
\hline Family Hx of CVD (Yes vs. No) & Total & & 2 & 51.1 & 2.04 & $1.94(0.93,4.06)^{\mathrm{OR}}$ & 0.077 \\
\hline \multirow{11}{*}{$\mathrm{BMI}\left(\mathrm{kg} / \mathrm{m}^{2}\right)$} & Total & & 4 & 79.9 & 14.96 & $0.05(-1.41,1.50)^{\mathrm{WMD}}$ & 0.949 \\
\hline & \multirow{2}{*}{ Type of control } & Healthy controls & 2 & 21.9 & 1.28 & $0.49(-0.32,1.31)^{\mathrm{WMD}}$ & 0.234 \\
\hline & & Hospital-based controls & 2 & 92.3 & 13.07 & $-0.83(-5.33,3.63)^{\mathrm{WMD}}$ & 0.709 \\
\hline & \multirow{3}{*}{ Type of stroke } & All & 1 & - & 0.00 & $-3.20(-5.25,-1.15)^{\mathrm{WMD}}$ & 0.002 \\
\hline & & IS & 3 & 23.4 & 2.61 & $0.70(-0.04,1.44)^{\mathrm{WMD}}$ & 0.062 \\
\hline & & $\mathrm{HS}$ & - & - & - & - & - \\
\hline & \multirow{3}{*}{ Study design } & Cohort & 2 & 21.9 & 1.28 & $0.49(-0.32,1.31)^{\mathrm{WMD}}$ & 0.234 \\
\hline & & Case-control & 2 & 92.3 & 13.07 & $-0.85(-5.33,3.63)^{\mathrm{WMD}}$ & 0.709 \\
\hline & & Cross-sectional & - & - & - & - & - \\
\hline & \multirow{2}{*}{ Matching } & Non-matching & 3 & 83.7 & 12.28 & $-0.43(-2.31,1.44)^{\mathrm{WMD}}$ & 0.649 \\
\hline & & Sex or age matching & 1 & - & 0.00 & $1.37(-0.03,2.77)^{\mathrm{WMD}}$ & 0.055 \\
\hline WC $(\mathrm{cm})$ & Total & & 3 & 27.7 & 2.77 & $3.25(1.44,5.06)^{\mathrm{WMD}}$ & $<0.001$ \\
\hline & Total & & 3 & 89.5 & 19.02 & $36.86(7.66,66.07)^{\mathrm{WMD}}$ & 0.013 \\
\hline & & Healthy controls & 2 & 0.0 & 0.73 & $15.78(7.61,23.96)^{\mathrm{WMD}}$ & $<0.001$ \\
\hline & Iype of control & Hospital-based controls & 1 & - & 0.00 & $104.49(64.66,144.32)^{\mathrm{WMD}}$ & $<0.001$ \\
\hline & & All & - & - & - & - & - \\
\hline & Type of stroke & IS & 3 & 89.5 & 19.02 & $36.86(7.66,66.07)^{\mathrm{WMD}}$ & 0.013 \\
\hline $\mathrm{FBG}(\mathrm{mg} / \mathrm{dL})$ & & $\mathrm{HS}$ & - & - & - & - & - \\
\hline & & Cohort & 2 & 0.0 & 0.73 & $15.78(7.61,23.96)^{\mathrm{WMD}}$ & $<0.001$ \\
\hline & Study design & Case-control & 1 & - & 0.00 & $104.49(64.66,144.32)^{\mathrm{WMD}}$ & $<0.001$ \\
\hline & & Cross-sectional & - & - & - & - & - \\
\hline & & Non-matching & 2 & 0.0 & 0.73 & $15.78(7.61,23.96)^{\mathrm{WMD}}$ & $<0.001$ \\
\hline & Matching & Sex or age matching & 1 & - & 0.00 & $104.49(64.66,144.32)^{\mathrm{WMD}}$ & $<0.001$ \\
\hline & Total & & 8 & 74.4 & 27.29 & $2.15(1.41,3.29)^{\mathrm{OR}}$ & $<0.001$ \\
\hline & Type of control & Healthy controls & 4 & 29.5 & 4.25 & $1.96(1.41,2.72)^{\mathrm{OR}}$ & $<0.001$ \\
\hline & Iype of control & Hospital-based controls & 4 & 86.9 & 22.98 & $2.54(1.01,6.43)^{\mathrm{OR}}$ & 0.049 \\
\hline & & All & - & - & - & - & - \\
\hline & Type of stroke & IS & 7 & 77.5 & 26.72 & $2.24(1.38,3.66)^{\mathrm{OR}}$ & 0.001 \\
\hline DM (Yes vs. No) & & $\mathrm{HS}$ & 1 & - & 0.00 & $1.69(0.97,2.94)^{\mathrm{OR}}$ & 0.066 \\
\hline & & Cohort & 2 & 25.6 & 1.34 & $2.29(1.57,3.35)^{\mathrm{OR}}$ & $<0.001$ \\
\hline & Study design & Case-control & 5 & 82.7 & 23.11 & $2.38(1.14,4.95)^{\mathrm{OR}}$ & 0.020 \\
\hline & & Cross-sectional & 1 & - & 0.00 & $1.23(0.65,2.32)^{\mathrm{OR}}$ & 0.518 \\
\hline & & Non-matching & 2 & 25.6 & 1.34 & $2.29(1.57,3.35)^{\mathrm{OR}}$ & $<0.001$ \\
\hline & Matchıng & Sex or age matching & 6 & 80.3 & 25.43 & $2.12(1.14,3.93)^{\mathrm{OR}}$ & 0.018 \\
\hline HTN (Yes vs. No) & Total & & 10 & 37.7 & 14.45 & $3.56(3.00,4.23)^{\mathrm{OR}}$ & $<0.001$ \\
\hline
\end{tabular}


Table 2. Continued

\begin{tabular}{|c|c|c|c|c|c|c|c|}
\hline Variables & & & $\mathbf{K}^{*}$ & $I^{2}(\%)$ & $Q$ test & Pooled ES $(95 \% \mathrm{CI})$ & $P$ Value \\
\hline $\mathrm{SBP}(\mathrm{mmHg})$ & Total & & 3 & 0.0 & 0.27 & $13.42(10.19,16.64)^{\mathrm{WMD}}$ & $<0.001$ \\
\hline $\mathrm{DBP}(\mathrm{mmHg})$ & Total & & 3 & 0.0 & 1.26 & $6.66(4.61,8.70)^{\mathrm{WMD}}$ & $<0.001$ \\
\hline \multirow{11}{*}{ Triglycerides (mg/dL) } & Total & & 8 & 60.9 & 17.88 & $21.48(10.03,32.94)^{\mathrm{WMD}}$ & $<0.001$ \\
\hline & \multirow{2}{*}{ Type of control } & Healthy controls & 4 & 55.6 & 6.76 & $24.48(10.15,38.81)^{\mathrm{WMD}}$ & 0.001 \\
\hline & & Hospital-based controls & 4 & 69.6 & 9.88 & $18.23(-1.68,38.14)^{\mathrm{WMD}}$ & 0.073 \\
\hline & \multirow{3}{*}{ Type of stroke } & All & - & - & - & - & - \\
\hline & & IS & 8 & 60.9 & 17.88 & $21.48(10.03,32.94)^{\mathrm{WMD}}$ & $<0.001$ \\
\hline & & $\mathrm{HS}$ & - & - & - & - & - \\
\hline & \multirow{3}{*}{ Study design } & Cohort & 2 & 0.0 & 0.29 & $10.85(-5.77,27.46)^{\mathrm{WMD}}$ & 0.201 \\
\hline & & Case-control & 5 & 73.1 & 14.86 & $23.99(7.46,40.51)^{\mathrm{WMD}}$ & 0.004 \\
\hline & & Cross-sectional & 1 & - & 0.00 & $24.20(3.60,44.80)^{\mathrm{WMD}}$ & 0.021 \\
\hline & \multirow{2}{*}{ Matching } & Non-matching & 3 & 0.0 & 1.27 & $16.11(3.18,29.04)^{\mathrm{WMD}}$ & 0.015 \\
\hline & & Sex or age matching & 5 & 73.1 & 14.86 & $23.99(7.46,40.51)^{\mathrm{WMD}}$ & 0.004 \\
\hline \multirow{11}{*}{$\mathrm{TC}(\mathrm{mg} / \mathrm{dL})$} & Total & & 6 & 86.9 & 30.06 & $15.08(0.48,29.68)^{\mathrm{WMD}}$ & 0.043 \\
\hline & \multirow{2}{*}{ Type of control } & Healthy controls & 3 & 93.9 & 32.67 & $18.79(-9.57,47.14)^{\mathrm{WMD}}$ & 0.194 \\
\hline & & Hospital-based controls & 3 & 46.8 & 3.76 & $11.92(-0.06,23.91)^{\mathrm{WMD}}$ & 0.051 \\
\hline & \multirow{3}{*}{ Type of stroke } & All & - & - & - & - & - \\
\hline & & IS & 6 & 86.9 & 30.06 & $15.08(0.48,29.68)^{\mathrm{WMD}}$ & 0.043 \\
\hline & & $\mathrm{HS}$ & - & - & - & - & - \\
\hline & \multirow{3}{*}{ Study design } & Cohort & 1 & - & 0.00 & $6.96(-3.20,17.12)^{\mathrm{WMD}}$ & 0.179 \\
\hline & & Case-control & 4 & 88.2 & 25.47 & $21.23(2.16,40.30)^{\mathrm{WMD}}$ & 0.029 \\
\hline & & Cross-sectional & 1 & - & 0.00 & $-2.80(-21.29,15.69)^{\mathrm{WMD}}$ & 0.767 \\
\hline & \multirow{2}{*}{ Matching } & Non-matching & 2 & 0.0 & 0.82 & $4.70(-4.20,13.60)^{\mathrm{WMD}}$ & 0.301 \\
\hline & & Sex or age matching & 4 & 88.2 & 25.47 & $21.23(2.16,40.30)^{\mathrm{WMD}}$ & 0.029 \\
\hline \multirow{11}{*}{ LDL-C (mg/dL) } & Total & & 5 & 94.9 & 77.83 & $23.89(0.93,46.84)^{\mathrm{WMD}}$ & 0.041 \\
\hline & \multirow{2}{*}{ Type of control } & Healthy controls & 2 & 97.3 & 37.58 & $21.33(-19.74,62.40)^{\mathrm{WMD}}$ & 0.309 \\
\hline & & Hospital-based controls & 3 & 95.0 & 39.95 & $25.86(-10.80,62.51)^{\mathrm{WMD}}$ & 0.167 \\
\hline & \multirow{3}{*}{ Type of stroke } & All & - & - & - & - & - \\
\hline & & IS & 5 & 94.9 & 77.83 & $23.89(0.93,46.84)^{\mathrm{WMD}}$ & 0.041 \\
\hline & & $\mathrm{HS}$ & - & - & - & - & - \\
\hline & \multirow{3}{*}{ Study design } & Cohort & 1 & - & 0.00 & $0.41(-8.75,9.57)^{\mathrm{WMD}}$ & 0.930 \\
\hline & & Case-control & 3 & 78.8 & 9.43 & $41.30(23.63,58.93)^{\mathrm{WMD}}$ & $<0.001$ \\
\hline & & Cross-sectional & 1 & - & 0.00 & $-2.10(-12.38,8.18)^{\mathrm{WMD}}$ & 0.689 \\
\hline & \multirow{2}{*}{ Matching } & Non-matching & 2 & 0.0 & 0.13 & $-0.70(-7.54,6.14)^{\mathrm{WMD}}$ & 0.841 \\
\hline & & Sex or age matching & 3 & 78.8 & 9.43 & $41.30(23.63,58.93)^{\mathrm{WMD}}$ & $<0.001$ \\
\hline \multirow{11}{*}{ HDL-C (mg/dL) } & \multicolumn{2}{|l|}{ Total } & 7 & 14.24 & 57.9 & $-2.31(-4.30,-0.33)^{\mathrm{WMD}}$ & 0.023 \\
\hline & \multirow[t]{2}{*}{ Type of control } & Healthy controls & 4 & 35.0 & 4.62 & $-0.79(-2.42,0.83)^{\mathrm{WMD}}$ & 0.337 \\
\hline & & Hospital-based controls & 3 & 0.0 & 1.21 & $-5.25(-8.05,-2.45)^{\mathrm{WMD}}$ & $<0.001$ \\
\hline & \multirow[t]{3}{*}{ Type of stroke } & All & - & - & - & - & - \\
\hline & & IS & 7 & 14.24 & 57.9 & $-2.31(-4.30,-0.33)^{\mathrm{WMD}}$ & 0.023 \\
\hline & & $\mathrm{HS}$ & - & - & - & - & - \\
\hline & \multirow[t]{3}{*}{ Study design } & Cohort & 2 & 0.0 & 0.04 & $0.17(-1.42,1.75)^{\mathrm{WMD}}$ & 0.838 \\
\hline & & Case-control & 4 & 6.8 & 3.22 & $-2.77(-4.67,-0.87)^{\mathrm{WMD}}$ & 0.004 \\
\hline & & Cross-sectional & 1 & - & 0.00 & $-6.90(-11.59,-2.21)^{\mathrm{WMD}}$ & 0.004 \\
\hline & \multirow{2}{*}{ Matching } & Non-matching & 3 & 74.5 & 7.85 & $-1.51(-4.75,1.73)^{\mathrm{WMD}}$ & 0.362 \\
\hline & & Sex or age matching & 4 & 6.8 & 3.22 & $-2.77(-4.67,-0.87)^{\mathrm{WMD}}$ & 0.004 \\
\hline
\end{tabular}

* K, number of included studies; ES, effect size; IS, Ischemic stroke; HS, Hemorrhage stroke; OR, odds ratio; WMD, weighted mean differences; BMI, body mass index; WC, waist circumference; FBG, fasting blood glucose; SBP, systolic blood pressure; DBP, diastolic blood pressure; TC, total-cholesterol; LDL-C, Low-density lipoprotein-cholesterol; HDL-c, High-density lipoprotein-cholesterol; DM, diabetes mellitus; HTN, hypertension. 
Table 3. Results of Sensitivity Analyses to Estimate the Effects of Each Study on Pooled ESs (WMD/OR)

\begin{tabular}{|c|c|c|c|c|c|c|c|}
\hline \multirow{2}{*}{ Variables } & \multicolumn{3}{|l|}{ Pre-sensitivity Analysis } & \multirow{2}{*}{$\begin{array}{l}\text { Upper \& } \\
\text { Lower of ES }\end{array}$} & \multicolumn{3}{|c|}{ Post-sensitivity Analysis } \\
\hline & No. of Studies Included & Pooled ESs & $95 \% \mathrm{Cl}$ & & Pooled ESs & $95 \% \mathrm{Cl}$ & Excluded Studies \\
\hline \multirow{2}{*}{ Mean age (year) } & \multirow{2}{*}{4} & \multirow{2}{*}{5.49} & \multirow{2}{*}{$4.45,6.54^{\mathrm{WMD}}$} & Upper & 6.79 & $5.25,8.33$ & Parizadeh et $\mathrm{al}^{20}$ \\
\hline & & & & Lower & 5.23 & $4.07,6.39$ & Sarrafzadegan et a $\left.\right|^{27}$ \\
\hline \multirow{2}{*}{ Sex (male vs. female) } & \multirow{2}{*}{6} & \multirow{2}{*}{1.23} & \multirow{2}{*}{$0.85,1.79^{\mathrm{OR}}$} & Upper & 1.38 & $1.09,1.76$ & Afshari et $\mathrm{al}^{28}$ \\
\hline & & & & Lower & 1.10 & $0.69,1.76$ & Saneei et $\mathrm{al}^{23}$ \\
\hline \multirow{2}{*}{ Smoking (Yes vs. no) } & \multirow{2}{*}{10} & \multirow{2}{*}{1.44} & \multirow{2}{*}{$1.02,2.03^{\mathrm{OR}}$} & Upper & 1.55 & $1.10,2.21$ & Hesami et al ${ }^{15}$ \\
\hline & & & & Lower & 1.29 & $0.93,1.79$ & Hamzehee-Moghadam et al ${ }^{29}$ \\
\hline \multirow{2}{*}{$\mathrm{BMI}\left(\mathrm{kg} / \mathrm{m}^{2}\right)$} & \multirow{2}{*}{4} & \multirow{2}{*}{0.05} & \multirow{2}{*}{$-1.41,1.50^{\mathrm{WMD}}$} & Upper & 0.70 & $-0.03,1.43$ & Saneei et $\mathrm{al}^{23}$ \\
\hline & & & & Lower & -0.43 & $-2.30,1.43$ & Moshayedi et al ${ }^{21}$ \\
\hline \multirow{2}{*}{ WC $(\mathrm{cm})$} & \multirow{2}{*}{3} & \multirow{2}{*}{3.25} & \multirow{2}{*}{$1.44,5.06 \mathrm{WMD}$} & Upper & 4.24 & $1.20,7.27$ & Parizadeh et $\mathrm{al}^{20}$ \\
\hline & & & & Lower & 2.97 & $0.74,5.21$ & Sarrafzadegan et al ${ }^{27}$ \\
\hline \multirow{2}{*}{$\mathrm{FBG}(\mathrm{mg} / \mathrm{dL})$} & \multirow{2}{*}{3} & \multirow{2}{*}{36.86} & \multirow{2}{*}{$7.66,66.07^{\text {WMD }}$} & Upper & 60.08 & $-22.54,142.70$ & Parizadeh et $\mathrm{a}^{20}$ \\
\hline & & & & Lower & 15.78 & $7.60,23.95$ & Afshari et $\mathrm{a}^{28}$ \\
\hline \multirow{2}{*}{ DM (Yes vs. No) } & \multirow{2}{*}{8} & 15 & $1 \quad 1120$ & Upper & 2.42 & $1.59,3.67$ & Ashjazadeh et $\mathrm{al}^{26}$ \\
\hline & & 2.15 & $1.41,3.290 \mathrm{~K}$ & Lower & 1.79 & $1.31,2.46$ & Moshayedi et $\mathrm{al}^{21}$ \\
\hline HTN Yoc Yc No) & 10 & 356 & 300 & Upper & 3.95 & $3.27,4.78$ & Parizadeh et $\mathrm{a}^{20}$ \\
\hline HIN (Yes vs. No) & 10 & 3.56 & $3.00,4.230 \mathrm{~K}$ & Lower & 3.30 & $2.74,3.99$ & Saneei et $\mathrm{al}^{23}$ \\
\hline $\mathrm{SRP}(\mathrm{mm} \mathrm{H} \sigma)$ & 2 & 1242 & $10-1664$ WMD & Upper & 14.07 & $10.01,18.12$ & Parizadeh et $\mathrm{a}^{20}$ \\
\hline SDF (IIII RI) & 3 & 13.42 & $10.19,10.64$ & Lower & 13.04 & $9.07,17.01$ & Sarrafzadegan et al ${ }^{27}$ \\
\hline & 2 & 666 & $1961=70$ WMD & Upper & 7.70 & $4.74,10.66$ & Parizadeh et al 20 \\
\hline DBP (mm Hg) & 3 & 6.66 & $4.61,8.70^{\text {Wun }}$ & Lower & 6.30 & $3.71,8.89$ & Sarrafzadegan et al ${ }^{27}$ \\
\hline 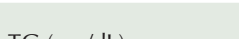 & 0 & 81 & (1) & Upper & 24.64 & $13.31,35.97$ & Savadi-Oskouei et al $^{31}$ \\
\hline IG (mg/dL) & 8 & 21.48 & $10.03,32.94^{\text {vim }}$ & Lower & 18.23 & $6.85,29.62$ & Hosinian et al ${ }^{16}$ \\
\hline & & & & Upper & 18.25 & $2.50,33.99$ & Maghbooli et al ${ }^{24}$ \\
\hline TC (mg/dL) & 6 & 15.08 & $0.48,29.68^{\mathrm{WMD}}$ & Lower & 14.37 & $-5.22,33.97$ & Savadi-Oskouei et $\mathrm{al}^{31}$ \\
\hline I $\mathrm{C}(\mathrm{mo} / \mathrm{d})$ & 5 & 2389 & 034684 WMD & Upper & 30.58 & $4.02,57.13$ & Maghbooli et $\mathrm{al}^{24}$ \\
\hline LDL-C (mg/aL) & 5 & 23.89 & $0.93,46.84$ & Lower & 15.54 & $-7.02,38.10$ & Moshayedi et $\mathrm{al}^{21}$ \\
\hline 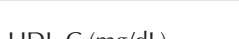 & 7 & 221 & 40 (1) 20 WMD & Upper & -1.50 & $-3.22,0.22$ & Maghbooli et al ${ }^{24}$ \\
\hline HDL-C (mg/dL) & 7 & -2.31 & $-4.30,-0.33$ wave & Lower & -3.06 & $-5.33,-0.78$ & Sarrafzadegan et $\mathrm{a}^{27}$ \\
\hline
\end{tabular}

BMI, Body mass index; WC, Waist circumference; FB, Fasting blood glucose; SBP, Systolic blood pressure; DBP, Diastolic blood pressure; TG, triglycerides; TC, Total-cholesterol; LDL-C, Low-density lipoprotein-cholesterol; HDL-C, High-density lipoprotein-cholesterol; DM, Diabetes mellitus; HTN, Hypertension.

\section{Discussion}

Stroke risk stratification depends on various regional and ethnic factors that are taken into account as integral parts of resource assessment as well as bases for developing targeted stroke prevention programs. Currently, there is lack of related literature on the Iranian population regarding the risk factors of either ischemic or hemorrhagic strokes. Thus, the present comprehensive meta-analysis of observational studies on the Iranian population as an example of other LMICs was conducted to explore SRFs among Iranians. Overall, the included observational studies covered demographics (age and gender), traditional VRFs (HTN, DM, dyslipidemia, and family history of CVDs), and lifestyle factors (CS, OD, WC, and BMI). Notably, the majority of these predisposing factors were preventable. This study also provided essential information and a global insight into all modifiable and non-modifiable SRFs in the Iranian population and added to the information of all previous epidemiological studies in this field. ${ }^{18,32-36}$ The meta-analysis findings also revealed that age, but not gender, was associated with the risk of stroke. HTN, SBP and DBP, DM, and FBG were accordingly correlated with increased risk of stroke among the traditional VRFs. Other possible risk factors including CS, OD, and WC were further confirmed as independent stroke determinants in addition to high circulating levels of TG, LDL-C, TC, and low HDL-C.

Demographics Factors

In this regard, a significant association was observed for mean age, whereas gender did not influence the risk of stroke. Stroke distribution was also correlated with age and gender. ${ }^{37}$ The highlighted impact of aging was in line with a previous systematic study from Iran, ${ }^{17}$ underlining a slight predominance for females in older age in contrast to a small prevalence for males in younger age. ${ }^{17}$ It should be noted that a large cohort study from northwestern Iran had reported a similar incidence in women and men. ${ }^{38}$ Nevertheless, in subgroup analyses, male gender was found to be significantly associated with stroke. However, these findings should be cautiously explored and further studies are needed to evaluate the rate of age-specific stroke in Iran because the number of articles in age-matched groups is not sufficient (only one study was age-matched for gender while five studies were not). Considering the fact that several studies from Middle Eastern countries such as Iraq, 
Table 4. The Findings of Quality Assessment Using the Newcastle-Ottawa Scale

\begin{tabular}{|c|c|c|c|c|c|}
\hline Author Name & Study design & Selection & Comparability & Exposure/Outcome & Overall Quality Assessment Score \\
\hline Fahimfar et al ${ }^{14}$ & Cohort sttudy & $* * * *$ & * & $* *$ & 7 \\
\hline Saneei et $\mathrm{al}^{23}$ & Case-control study & $* *$ & $* *$ & $* *$ & 6 \\
\hline Moshayedi et $\mathrm{al}^{21}$ & Case-control study & $* * *$ & * & ** & 6 \\
\hline Maghbooli et $\mathrm{al}^{24}$ & Cross-sectional study & $* * *$ & $* *$ & $* *$ & 7 \\
\hline Sadreddini et $\mathrm{al}^{25}$ & Case-control study & $* * *$ & * & $* * *$ & 7 \\
\hline Parizadeh et $\mathrm{al}^{20}$ & Cohort Study & $* * *$ & $* *$ & $* * *$ & 8 \\
\hline Hosinian et $\mathrm{al}^{16}$ & Case-control study & $* *$ & * & ** & 5 \\
\hline Ashjazadeh et $\mathrm{al}^{26}$ & Case-control study & $* *$ & * & $* * *$ & 6 \\
\hline Hesami et al ${ }^{15}$ & Case-control study & $* *$ & * & ** & 5 \\
\hline Sarrafzadegan et $\mathrm{al}^{27}$ & Cohort study & $* * * *$ & * & $* *$ & 7 \\
\hline Saberi et $\mathrm{a}^{22}$ & Cross-sectional study & $* *$ & * & * & 4 \\
\hline Afshari et $a^{28}$ & Case-control study & *** & ** & ** & 7 \\
\hline Hamzehee-Moghadam et $\mathrm{al}^{29}$ & Case-control study & $* *$ & * & $* * *$ & 6 \\
\hline Savadi-Oskouei et $\mathrm{al}^{30}$ & Case-control study & * & * & $* *$ & 4 \\
\hline Savadi-Oskouei et $\mathrm{al}^{31}$ & Case-control study & * & * & $* *$ & 4 \\
\hline
\end{tabular}

* For case-control studies, there are four items within the selection: 1) indicates cases adequately defined; 2) representativeness of the series of cases; 3) community controls; 4) controls have a group of stroke-free population), one item (with two sub-items A and B) within the comparability: A study controls for important factor and B study controls for additional factor(s), and three items within the exposure: 1) ascertainment of exposure by records; 2) same method of ascertainment used for cases and controls; and 3) same non-response rate for both groups. For cohort studies are four items within the selection: 1 ) shows exposed cohort truly representative in the studied community; 2) non-exposed cohort drawn from the same community as the exposed cohort; 3) ascertainment of exposure by records; 4) outcome of interest was not present at start of study, one item (with two sub-items A and B) within the comparability: A cohorts comparable on the basis of important factor and B cohorts comparable for any additional factor, and three items within the outcome: 1) assessment of outcome by record linkage or independent blinding; 2), follow-up long enough for outcomes to occur; and 3) follow up adequacy of cohorts. For cross-sectional studies are three items within the selection: 1) shows samples truly representative; 2) same characteristics between respondents and non-respondents and enough response rate; 3 ) exposure ascertainment by validated measurement tool, one item (with two sub-items A and B) within the comparability: A study controls for important factor and B study controls for additional factor, and two items within the outcome: 1) assessment of outcome by record linkage or independent blinding; 2) the statistical test is clearly described and appropriately presented.

Saudi Arabia, and Palestine have shown significant female predominance, ${ }^{39}$ determining gender-related predisposing factors requires in-depth investigations to shed light on the causes of this discrepancy between Iran and other nations in the Middle East.

\section{Traditional VRFs}

To examine the metabolic profile of the patients, the crucial role of diet should be taken into account. Diet can be thus involved in numerous interconnected mechanisms underlying stroke and related to balance in blood pressure, blood lipids, thrombosis and coagulation, oxidative stress, systemic inflammation (SI), endothelial function, glucose/ insulin homeostasis, gut microbiome, and body weight. ${ }^{40}$ HTN, DM, and dyslipidemia (including high levels of TG, TC, LDL-C, and low levels of HDL-C), as three comorbidities that could very often co-exist in patients, were thus regarded as SRFs in this study. It seems that these populations are susceptible to higher TG and lower HDL-C levels. Nevertheless, after additional analysis based on gender and age matching, the findings revealed that TC and LDL-C levels were also significantly associated as SRFs. A meta-analysis by Wang et al further concluded that a higher level of HDL-C was correlated with a lower risk of hemorrhagic stroke. ${ }^{41}$ Of note, overall dyslipidemia is one of the important risk factors for coronary artery diseases (CADs) and stroke, which can be affected and changed by lifestyle, diet, and medications. ${ }^{42}$ In this respect, recent studies have suggested that inadequate physical activity is correlated with decreased HDL-C and increased TG levels. ${ }^{43,44}$ Also, HTN and DM have been repeatedly among the strongest SRFs. ${ }^{45-47} \mathrm{DM}$ also augments the risk of stroke by four times. ${ }^{48}$ The INTERSTROKE study had similarly listed HTN and DM among the ten factors accounting for over $90 \%$ of SRFs, ${ }^{10}$ which is consistent with the results of a nationwide German study focusing on stroke onset below 55 years of age. In that study, HTN, dyslipidemia, and DM were mentioned among the top eight risk factors and the similarity with attributed risks in the older population was emphasized. ${ }^{49} \mathrm{~A}$ meta-analysis of the Chinese population correspondingly underlined the significance of HTN and DM as leading SRFs while hyperlipidemia was mildly correlated. ${ }^{47} \mathrm{~A}$ review of the Middle East studies on stroke patients further revealed that the prevalence rate of HTN was $62.1 \%$ followed by dyslipidemia with $36.8 \%$ and DM with $33.1 \% .{ }^{18}$ Although many risk factors are common across demographic groups, a universal weight cannot be applied due to variations in importance and prevalence of the given factors. ${ }^{10,47}$ Thus, screening and prevention should be designated based on local priorities. However, a holistic approach is crucial as risk factors are often intertwined. Furthermore, LMICs should assess what part of resources, i.e., human resources, healthcare facilities, diagnostic and laboratory 
services, medications, and access to transportation, is more important to be improved with regard to stroke care..$^{50}$

In this study, family history of CVDs was not significantly correlated with stroke in the Iranian population. This might be due to the limitation in the included studies that had been considered suitable for evaluating the relationship between family history and stroke. It should be noted that family history is a well-established $S R F^{51}$ especially at a younger age. ${ }^{33,52}$ In this respect, a national cohort study in the United States showed that a positive family history of stroke was associated with physiological and behavioral factors such as HTN and CS, implying that family history could be also partially attributed to modifiable factors. ${ }^{53}$ Unfortunately, the presence of such an association could not be assessed based on the existing data in this study. Accordingly, encouraging family members of stroke patients to pursue a stricter healthy lifestyle and to adhere to routine monitoring seems very reasonable principally in LMICs, wherein prevention should be highly prioritized due to limited resources.

\section{Lifestyle Factors}

Extensive research has revealed the role of CS in stroke. ${ }^{10,46,49}$ A meta-analysis covering studies for almost 50 years on approximately four million individuals also confirmed CS as an independent SRF with a similar risk in both men and women, confirming that both genders could benefit from CS cessation. ${ }^{54}$ Moreover, non-smokers, especially women, could be subject to a greater risk of stroke, ischemic heart disease, and chronic obstructive pulmonary disease (COPD) subsequent to second-hand smoke exposure. ${ }^{55}$ Accordingly, Austin et al ${ }^{56}$ suggested that the link between COPD and stroke was not only correlated with shared traditional risk factors including aging and smoking, but also the COPD-related SI and oxidative stress that could aggravate cerebral vascular dysfunction and platelet hyperactivity.

Regarding dependence, most studies have focused on alcohol abuse, while it is less prevalent in Iran and very likely to be underreported in surveys due to its illegality. As a result, no assumptions could be offered on alcohol. On the other hand, OD is a more common public health concern in Iran. It is noteworthy that substance abuse is illegal in Iran and it is socially stigmatized. Therefore, the possibility of underreporting cannot be ignored. The findings revealed that total OD (regardless of its type) was a SRF. Several studies on the Iranian population have also confirmed OD as a risk factor for stroke and CVDs, ${ }^{13,57-59}$ possibly by enhancing plasma fibrinogen activity and chance of atherosclerosis. ${ }^{59}$

The impact of obesity and BMI on development of stroke has been similarly supported in large-scale studies. ${ }^{49} \mathrm{~A}$ meta-analysis by Guo et $\mathrm{al}^{60}$ concluded that young adults with overweight or obesity were prone to an elevated risk of stroke probably independent of other
cardioVRFs. A meta-analysis of prospective studies with two million participants by Strazzullo et al further found that overweight and obesity were linked with increased risk of ischemic stroke, at least in part, independently from lifestyle, age, and other VRFs. ${ }^{61}$ The findings of the present study were in favor of the adverse impact of WC on stroke but not BMI. Interestingly, it has been demonstrated that by excluding the effect of BMI, abdominal obesity per $s e$, in terms of WC and waist-to-height ratio, turns out to be a common risk factor in both genders. ${ }^{62}$ Further studies are thus needed to assess the role of WC as a SRF in comparison with other anthropometric factors such as BMI. Unfortunately, the infrastructure to facilitate physical activity is rather underdeveloped in LMICs like Iran, even though the costs can outweigh the damage from diseases related to excess weight and sedentary life.

\section{Strengths and Limitations}

The present study was the first attempt as the most comprehensive systematic review and meta-analysis to determine SRF in the Iranian population. However, there were several limitations. Firstly, the number of studies evaluated for risk factors affecting stroke in the Iranian population was small. Secondly, some articles were excluded due to their methodological limitations. Thirdly, there was heterogeneity among the included primary studies regarding their outcomes. Fourthly, the subgroup analysis did not adjust for confounding variables. Therefore, the results are possibly affected by confounding factors and should be interpreted with caution. Accordingly, the relationship between each risk factor and stroke was estimated based on a comparison of the distribution of risk factors between case and control groups (univariate analysis) because the studies with the adjusted findings were not adequate.

\section{Conclusion}

Apparently, stroke seems to be strongly associated with lifestyle and environmental age-related factors in Iran. Among the risk factors mentioned, HTN, SBP and DBP, $\mathrm{DM}$, and FBG were related to increased risk of stroke among traditional VRFs. Other possible risk factors including $\mathrm{CS}$, OD, and WC were further established as independent stroke determinants as well as high circulating levels of TG, LDL-C, TC, and low HDL-C. Considering recent lifestyle alterations in the Iranian society, stroke continues to take its toll on a more extensive scale, especially with a shift towards the younger population if prompt public awareness and strict preventive policies remain neglected.

\section{Authors' Contribution}

$\mathrm{RT}, \mathrm{KBL}$ and, $\mathrm{ABH}$ designed the study. RT and $\mathrm{BK}$ participated in statistical analysis. RT, KBL, BK, HA, MRA, ZA, and ABH drafted and critically revised the manuscript.

Data Sharing Statement

No additional data are available. 
Conflict of Interest Disclosures

None declared.

\section{Ethical Statement}

Not applicable.

\section{Funding}

The research grant provided by the National Institutes for Medical Research Development (NIMAD) with number: 977269, Tehran, Iran.

\section{References}

1. Global, regional, and national burden of neurological disorders during 1990-2015: a systematic analysis for the Global Burden of Disease Study 2015. Lancet Neurol. 2017;16(11):877-97. doi: 10.1016/s1474-4422(17)30299-5.

2. Feigin VL, Norrving B, Mensah GA. Global Burden of Stroke. Circ Res. 2017;120(3):439-48. doi: 10.1161/ circresaha.116.308413.

3. Salter K, Zettler L, Foley N, Teasell R. Impact of caring for individuals with stroke on perceived physical health of informal caregivers. Disabil Rehabil. 2010;32(4):273-81. doi: 10.3109/09638280903114394

4. Dalvandi A, Ekman SL, Maddah SSB, Khankeh HR, Heikkilä K. Post Stroke life in Iranian people: used and recommended strategies. Iranian Rehabilitation Journal 2009;7(1):17-24.

5. Feigin VL, Forouzanfar $M H$, Krishnamurthi $R$, Mensah GA, Connor M, Bennett DA, et al. Global and regional burden of stroke during 1990-2010: findings from the Global Burden of Disease Study 2010. Lancet. 2014;383(9913):245-54. doi: 10.1016/s0140-6736(13)61953-4.

6. Kim AS, Cahill E, Cheng NT. Global stroke belt: geographic variation instrokeburden worldwide.Stroke. 2015;46(12):356470. doi: 10.1161/STROKEAHA.115.008226.

7. Feigin VL, Krishnamurthi R, Bhattacharjee R, Parmar P, Theadom A, Hussein T, et al. New strategy to reduce the global burden of stroke. Stroke. 2015;46(6):1740-7. doi: 10.1161/ STROKEAHA.115.008222

8. O'Donnell M, Yusuf S. Tackling the global burden of stroke: the need for large-scale international studies. Lancet Neurol. 2009;8(4):306-7. doi: 10.1016/s1474-4422(09)70024-9

9. Feigin VL, Krishnamurthi RV, Parmar P, Norrving B, AM George, Bennett DA, et al. Update on the Global Burden of Ischemic and Hemorrhagic Stroke in 1990-2013: The GBD 2013 Study. Neuroepidemiology 2015;45(3):161-76. doi: 10.1159/000441085

10. O'Donnell MJ, Chin SL, Rangarajan S, Xavier D, Liu L, Zhang $\mathrm{H}$, et al. Global and regional effects of potentially modifiable risk factors associated with acute stroke in 32 countries (INTERSTROKE): a case-control study. Lancet. 2016;388(10046):761-75. doi: 10.1016/s01406736(16)30506-2

11. O'Donnell MJ, Xavier D, Liu L, Zhang H, Chin SL, RaoMelacini P, et al. Risk factors for ischaemic and intracerebral haemorrhagic stroke in 22 countries (the INTERSTROKE study): a case-control study. Lancet. 2010;376(9735):112-23. doi: 10.1016/s0140-6736(10)60834-3

12. Azarpazhooh MR, Etemadi MM, Donnan GA, Mokhber N, Majdi MR, Ghayour-Mobarhan M, et al. Excessive incidence of stroke in Iran: evidence from the Mashhad Stroke Incidence Study (MSIS), a population-based study of stroke in the Middle East. Stroke. 2010;41(1):e3-e10. doi: 10.1161/ STROKEAHA.109.559708

13. Ebrahimi $H$, Haghjoo Javanmard $S$, Asgary $S$, Dehghani $L$, Amiri $M$, Saadatnia $M$, et al. Opium Addiction and Ischemic Stroke in Isfahan, Iran: A Case-Control Study. Eur Neurol. 2018;79(1-2):82-85. doi: 10.1159/000485098

14. Fahimfar N, Khalili D, Mohebi R, Azizi F, Hadaegh F. Risk factors for ischemic stroke; results from 9 years of follow-up in a population based cohort of Iran. BMC Neurol. 2012;12:117. doi: 10.1186/1471-2377-12-117

15. Hesami O, Kasmaei HD, Matini F, Assarzadegan F, Mansouri B, Jabbehdari $S$. Relationship between intracerebral hemorrhage and diabetes mellitus: a case-control study. J Clin Diagn Res. 2015;9(4):OC08-10. doi: 10.7860/JCDR/2015/12226.3741

16. Hosinian M, Qujeq D, Ahmadi Ahangar A. The Relation Between GABA and L-Arginine Levels With Some Stroke Risk Factors in Acute Ischemic Stroke Patients. Int J Mol Cell Med. 2016;5(2):100-5.

17. Hosseini AA, Sobhani-Rad D, Ghandehari K,Benamer $\mathrm{HT}$. Frequency and clinical patterns of stroke in Iran Systematic and critical review. BMC Neurol. 2010;10:72. doi: 10.1186/1471-2377-10-72

18. Behrouz R, Powers CJ. Epidemiology of classical risk factors in stroke patients in the Middle East. Eur J Neurol. 2016;23(2):262-9. doi: 10.1111/ene.12742

19. Borhani Haghighi A, Karimi AA, Amiri A, Ghaffarpasand F. Knowledge and attitude towards stroke risk factors, warning symptoms and treatment in an Iranian population. Med Princ Pract. 2010;19(6):468-72. doi: 10.1159/000320306

20. Parizadeh D, Ramezankhani A, Momenan AA, Azizi F, Hadaegh F. Exploring risk patterns for incident ischemic stroke during more than a decade of follow-up: A survival tree analysis. Comput Methods Programs Biomed. 2017;147:2936. doi: 10.1016/j.cmpb.2017.06.006

21. Moshayedi H, Ahrabi R, Mardani A, Sadigetegad S, Farhudi M. Association between non-alcoholic fatty liver disease and ischemic stroke. Iran J Neurol. 2014;13(3):144-8.

22. Saberi A, Ghayeghran AR, Janeshin S, Biazar G, Kazemnezhad E. Opium Consumption Prevalence Among Patients With Ischemic Stroke Compared With Healthy Individuals in Iran. Int J High Risk Behav Addict. 2017;6(1):e27264. doi: 10.5812/ ijhrba. 27264

23. Saneei P, Saadatnia M, Shakeri F, Beykverdi M, Keshteli $\mathrm{AH}$, Esmaillzadeh A. A case-control study on red meat consumption and risk of stroke among a group of Iranian adults. Public Health Nutr. 2015;18(6):1084-90. doi: 10.1017/ S1368980014001165.

24. Maghbooli M, Farhadi S, Esmaeilzadeh A, Mazloumzadeh S. Assessment of serum uric acid and vitamin $C$ levels in acute atherothrombotic stroke [in Persian]. ZUMS Journal. 2012;20(81):64-74.

25. Sadreddini SA, Abolfathi AA, Khandagi R, Talebi M, Lakian A. C-reactive protein, fibrinogen, lipoprotein (a), and lipid profile levels and platelet counts in ischemic stroke patients. Neurosciences (Riyadh). 2007;12(3):202-6.

26. Ashjazadeh N, Fathi M, Shariat A. Evaluation of Homocysteine Level as a Risk Factor among Patients with Ischemic Stroke and Its Subtypes. Iran J Med Sci. 2013;38(3):233-9.

27. Sarrafzadegan N, Gharipour M, Sadeghi M, Nezafati P, Talaie $\mathrm{M}$, Oveisgharan S, et al. Metabolic syndrome and the risk of ischemic stroke. J Stroke Cerebrovasc Dis. 2017;26(2):286-94. doi: 10.1016/j.jstrokecerebrovasdis.2016.09.019.

28. Afshari L, Amani R, Soltani F, Haghighizadeh MH, Afsharmanesh MR. The relation between serum Vitamin D levels and body antioxidant status in ischemic stroke patients: A case-control study. Adv Biomed Res. 2015;4:213. doi: 10.4103/2277-9175.166150.

29. Hamzehee Moghadam A, Mousavi A. The relationship between opium dependency and stroke [in Persian]. J Kerman Univ Medical Sci. 2006(4):203-08.

30. Savadi Oskoui O, Aminisani N, Hashemilar M. Major Risk Factors of Ischemic Stork in Women, 2002 [in Persian]. J Ardabil Univ Med Sci. 2003;3(1):23-28.

31. Savadi Oskoui D, Aminisani N, Hashemilar M. Blood Lipids and Ischemic Stroke: A Case Control Study [in Persian]. J Ardabil Univ Med Sci. 2004;3(4):27-31. 
32. Daneshfard B, Izadi S, Shariat A, Toudaji MA, Beyzavi Z, Niknam L. Epidemiology of stroke in Shiraz, Iran. Iran J Neurol. 2015;14(3):158-63.

33. Lutski M, Zucker I, Shohat T, Tanne D. Characteristics and Outcomes of Young Patients with First-Ever Ischemic Stroke Compared to Older Patients: The National Acute Stroke ISraeli Registry. Front Neurol. 2017;8:421. doi: 10.3389/ fneur.2017.00421.

34. Masoud SA, Dastmalchi F, Moosavi GA, Daneshvar-Kakhaki $\mathrm{R}$. Evaluating the relationship between highly sensitive $-\mathrm{C}$ reactive protein and acute cerebral ischemia [in Persian]. Feyz 2012;16(5):439-44.

35. Mohamadi S, Motamed N, Ostovar A,Nemati R. Epidemiology of stroke in the south west of Iran: A cohort study. Soc Determ Health. 2017;3(4):186-94. doi:10.22037/sdh.v3i4.19188.

36. Oygarden H, Fromm A, Sand KM, Kvistad CE, Eide GE, Thomassen L, et al. A Family History of Stroke Is Associated with Increased Intima-Media Thickness in Young Ischemic Stroke - The Norwegian Stroke in the Young Study (NORSYS). PloS One. 2016;11(8):e0159811. doi: 10.1371/journal. pone. 0159811.

37. Liberale L, Carbone F, Montecucco F, Gebhard C, Lüscher TF, Wegener S, et al. Ischemic stroke across sexes: What is the status quo? Front Neuroendocrinol. 2018. doi: 10.1016/j. yfrne.2018.05.001

38. Amiri A, Kapral MK, Thrift AG, Sposato LA, Saber H, Behrouz $\mathrm{R}$, et al. The Incidence and Characteristics of Stroke in Urban-Dwelling Iranian Women. J Stroke Cerebrovasc Dis. 2018;27(3):547-54. doi: 10.1016/j. jstrokecerebrovasdis.2017.09.050.

39. El-Hajj M, Salameh P, Rachidi S, Hosseini H. The epidemiology of stroke in the Middle East. Eur Stroke J. 2016;1(3):180-98. doi: $10.1177 / 2396987316654338$

40. Larsson SC. Dietary Approaches for Stroke Prevention. Stroke. 2017;48(10):2905-11. doi: 10.1161/strokeaha.117.017383

41. Wang X, Dong Y, Qi X, Huang C, Hou L. Cholesterol levels and risk of hemorrhagic stroke: a systematic review and meta-analysis. Stroke. 2013;44(7):1833-9. doi: 10.1161/ STROKEAHA. 113.001326

42. Tziomalos K, Athyros VG, Karagiannis A, Mikhailidis DP. Dyslipidemia as a risk factor for ischemic stroke. Curr Top Med Chem. 2009;9(14):1291-7. doi: $10.2174 / 156802609789869628$

43. O'Donovan G, Stensel D, Hamer M, Stamatakis E. The association between leisure-time physical activity, low HDL-cholesterol and mortality in a pooled analysis of nine population-based cohorts. Eur J Epidemiol. 2017;32(7):55966. doi: 10.1007/s10654-017-0280-9.

44. Jan CF, Chang HC, Tantoh DM, Chen PH, Liu WH, Huang JY. Duration-response association between exercise and HDL in both male and female Taiwanese adults aged 40 years and above. Oncotarget. 2018;9(2):2120-27. doi: 10.18632/ oncotarget.23251.

45. Alloubani A, Saleh A, Abdelhafiz I. Hypertension and diabetes mellitus as a predictive risk factors for stroke. Diabetes Metab Syndr. 2018;12(4):577-84. doi: 10.1016/j.dsx.2018.03.009.

46. Venketasubramanian N, Yoon BW, Pandian J, Navarro JC. Stroke Epidemiology in South, East, and South-East Asia: A Review. J Dtroke. 2017;19(3):286-94. doi: 10.5853/ jos.2017.00234

47. Wang J, Wen X, Li W, Li X, Wang Y, Lu W. Risk factors for stroke in the chinese population: a systematic review and meta-analysis. J Stroke Cerebrovasc Dis. 2017;26(3):509-17. doi: $\quad$ 10.1016/j.jstrokecerebrovasdis.2016.12.002

48. Tun NN, Arunagirinathan G, Munshi SK, Pappachan JM.
Diabetes mellitus and stroke: A clinical update. World J Diabetes. 2017;8(6):235-48. doi: 10.4239/wjd.v8.i6.235.

49. Aigner A, Grittner U, Rolfs A, Norrving B, Siegerink B, Busch MA. Contribution of Established Stroke Risk Factors to the Burden of Stroke in Young Adults. Stroke. 2017;48(7):174451. doi: 10.1161/strokeaha.117.016599.

50. Khatib R, Jawaada AM, Arevalo YA, Hamed HK, Mohammed $\mathrm{SH}$, Huffman MD. Implementing Evidence-Based Practices for Acute Stroke Care in Low-and Middle-Income Countries. Curr Atheroscler Rep. 2017;19(12):61. doi: 10.1007/s11883-0170694-6.

51. Park HJ, Kim TU, Hyun JK, Kim JY. Family history and functional outcome in Korean stroke patients: a preliminary study. Ann Rehabil Med. 2015;39(6):980-5. doi: 10.5535/ arm.2015.39.6.980

52. Jimenez-Gonzalez MC, Santiago-German D, CastilloHenkel EF, Alvarado-Moreno JA, Hernández-Juárez J, Leaños-Miranda A, et al. Identification of genetic risk factors associated with ischaemic stroke in young Mexican patients. Neurologia. 2018;S0213-4853(18)30032-X. . doi: 10.1016/j. $\mathrm{nrl} .2018 .01 .010$

53. Kulshreshtha A, Vaccarino V, Goyal A, McClellan W, Nahab F, Howard VJ, et al. Family history of stroke and cardiovascular health in a national cohort. J Stroke Cerebrovasc Dis. 2015;24(2):447-54. doi: 10.1016/j. jstrokecerebrovasdis.2014.09.017.

54. Peters SA, Huxley RR, Woodward M. Smoking as a risk factor for stroke in women compared with men: a systematic review and meta-analysis of 81 cohorts, including 3,980,359 individuals and 42,401 strokes. Stroke. 2013;44(10):2821-8. doi: 10.1161/strokeaha.113.002342.

55. Fischer F, Kraemer A. Meta-analysis of the association between second-hand smoke exposure and ischaemic heart diseases, COPD and stroke. BMC Public Health. 2015;15:1202. doi: 10.1186/s12889-015-2489-4.

56. Austin V, Crack PJ, Bozinovski S, Miller AA, Vlahos R. COPD and stroke: are systemic inflammation and oxidative stress the missing links? Clin Sci (Lond). 2016;130(13):1039-50. doi: 10.1042/cs20160043

57. Fallahzadeh MA, Salehi A, Naghshvarian M, Fallahzadeh MH, Poustchi H, Sepanlou SG, et al. Epidemiologic Study of Opium Use in Pars Cohort Study: A Study of 9000 Adults in a Rural Southern Area of Iran. Arch Iran Med. 2017;20(4):205-10.

58. Khademi H, Malekzadeh R, Pourshams A, Jafari E, Salahi R, Semnani S, et al. Opium use and mortality in Golestan Cohort Study: prospective cohort study of 50,000 adults in Iran. BMJ. 2012;344:e2502. doi: 10.1136/bmj.e2502

59. Hamzei Moqaddam A, Ahmadi Musavi SM, Khademizadeh K. Relationship of opium dependency and stroke. Addict Health. 2009;1(1):6-10.

60. GuoY, YueXJ, LiHH, Song ZX, Yan HQ, Zhang P, etal. Overweight and Obesity in Young Adulthood and the Risk of Stroke: a Meta-analysis. J Stroke Cerebrovasc Dis. 2016;25(12):29953004. doi: 10.1016/j.jstrokecerebrovasdis.2016.08.018.

61. Strazzullo P, D'Elia L, Cairella G, Garbagnati F, Cappuccio FP, Scalfi L. Excess body weight and incidence of stroke: meta-analysis of prospective studies with 2 million participants. Stroke. 2010;41(5):e418-26. doi: 10.1161/ STROKEAHA.109.576967.

62. Rodriguez-Campello A, Jimenez-Conde J, Ois A, CuadradoGodia E, Giralt-Steinhauer E, Vivanco RM, et al. Sex-related differences in abdominal obesity impact on ischemic stroke risk. Eur J Neurol. 2017;24(2):397-403. doi: 10.1111/ ene. 13216 